\title{
Embolic stroke of undetermined source in a young female with a history of intravenous drug use
}

\author{
Gabrielle Hayduk-Costa BSc \\ ${ }^{\prime}$ Class of 2017, Faculty of Medicine, Dalhousie University
}

\begin{abstract}
Care for patients with stroke depends on individual factors, including appropriateness of administering tissue plasminogen activator (tPA), patient age, and causational factors contributing to the stroke. We present a 52-yearold patient who, due to poor intravenous access, could not receive tPA. Despite multiple investigations, no obvious mechanistic cause for her stroke could be found, and she can therefore be classified within the relatively new construct of embolic stroke of undetermined source. The implications of this on the patient's care are discussed, along with a brief review of the literature.
\end{abstract}

42-year-old Caucasian woman presented to the Aemergency department after having fallen in the early morning. She had been watching television but upon standing to go back to bed, fell; this occurred shortly after 05:00. The fall was unwitnessed and therefore there was no collateral history of seizure activity, though the patient believed she lost consciousness. She was unable to move her left side, and was found on the floor by her partner, who brought her to the emergency department where she was seen two hours after her fall. She reported having felt unwell with nausea and vomiting about one week prior for three days.

Physical examination showed left hemiparesis including a left facial droop, with a left visual field defect and right gaze preference. Her National Institutes of Health Stroke Scale (NIHSS) was not calculated upon her arrival, but based on available information was between 10 and 19, placing her stroke in the moderate to severe category. ${ }^{1}$ Her initial measures were otherwise normal: she scored a 15/15 on the Glasgow Coma Scale, appeared to have normal motor speech, expressive language, and comprehension. She could answer simple questions and follow simple commands.

A non-contrast head computerized tomography (CT) scan showed evidence of two previous rightsided frontal lobe infarcts with no evidence of acute hemorrhage. The patient was found to be eligible for tissue plasminogen activator (tPA), but due to multiple failed attempts at intravenous (IV) access, including an attempt to place a femoral vein catheter, the drug could not be administered. The patient's poor IV access was thought to be related to a history of IV drug use.

Magnetic resonance imaging (MRI) revealed a right middle cerebral artery (MCA) infarct with midline shift due to occlusion of the M1 segment, which was consistent with subsequent findings on magnetic resonance angiography (MRA). MRA showed normalappearing intracranial arteries otherwise.

Attempts at uncovering the etiology of the stroke, which was believed to be embolic, were unsuccessful. Transthoracic echocardiogram (TTE) and transesophageal echocardiogram (TEE) showed no obvious abnormalities, and no cardiac source of emboli. Routine Holter monitoring showed normal sinus rhythm with no sustained arrhythmias. A carotid Doppler was normal. Hypercoagulability and vasculitis panels were unremarkable, with the exception of mildly low antithrombin III and protein C, the significance of which will be elaborated upon in the discussion. There was no evidence of recent IV drug use or endocarditis, though a drug screen was not done at the time of patient presentation.

\section{Discussion}

Many facets of this patient's presenting problem and subsequent care were unusual. She was not in the usual age range for stroke, and can be considered to fall within the category of stroke in the young. Given that the source of her embolic stroke was uncertain, the new clinical construct of embolic stroke of undetermined source (ESUS) applies to her. This patient would likely have benefitted from tPA, which could not be administered due to poor venous access. The implications of this latter point will not be discussed within this case study.

\section{Stroke in the young}

Ischemic stroke in the young accounts for a small percentage of total cases of stroke, and involves unique factors contributing both to the stroke itself and to its subsequent management. While definitions vary of what constitutes "stroke in the young", with many studies using an age limit near 45 years of age, studies aiming to be more inclusive may use limits up to 55 years of 
age..$^{2-4}$ Regardless of age, strokes can be devastating to the person and family. This may be particularly true in younger patients who often are responsible for caring and generating income for their families, and who lose a higher number of productive years. ${ }^{5}$

While prominent risk factors for stroke remain pertinent in the young (hypertension, cigarette smoking, hyperlipidemia, diabetes mellitus, and family history of stroke), some risk factors play a more important role in the young than they do in older populations, such as use of oral contraceptives, migraine, trauma, pregnancy or puerperium, and use of illicit drugs. ${ }^{2,4}$ Causes also differ between populations: strokes are more often caused by arterial dissection and cardioembolic etiologies in younger patients in developed countries, as opposed to atherosclerosis in older patients. ${ }^{3}$ Unfortunately, the etiology of strokes in the young is often undiscovered. Though reports differ, perhaps complicated by the varying definitions of what constitutes stroke in the "young" and variable intensity of investigations (e.g., more liberal use of TEE would likely uncover a higher predominance of patients with a patent foramen ovale [PFO]), stroke etiology remains unknown in roughly one third of young patients. ${ }^{2,3,5}$ This proportion tends to decrease with age. ${ }^{4}$ The uncertainty creates a challenge for patient management, as control of risk factors and decreasing the risk of subsequent events (i.e., secondary prevention) is ideally targeted towards etiologically related factors. Controlling well-known stroke risk factors is nonetheless important. As such, the patient discussed here was prescribed a statin for dyslipidemia, which was discovered in hospital. Her blood pressure and glucose were within normal ranges and she had quit smoking two years prior.

A history of illicit drug use is potentially an important contributing factor of stroke in young adults. ${ }^{6}$ Specifically, cocaine use has been shown to be associated with an increased risk of both hemorrhagic and ischemic stroke, with hypotheses around mechanisms including cerebral vasoconstriction, inflammatory changes to vasculature, endothelial dysfunction, and rupture of existing cerebral aneurysms due to sudden increases in blood pressure. ${ }^{7}$ However, a recent systematic review was equivocal, as a relationship between cocaine use and hemorrhagic (both subarachnoid and intracerebral) and ischemic stroke was not consistently supported by the studies examined. ${ }^{7}$ This topic of research is fraught with confounding factors, including gender, race/ethnicity, use of other illicit drugs, smoking, socioeconomic factors, accessibility to the health care system, risky lifestyle, other comorbidities (e.g., diabetes, hypertension, hyperlipidemia, etc.), use of contraceptives, human immune deficiency virus infection, and specifics around cocaine use (dosage, frequency, recency). ${ }^{7}$ Given the difficulty of controlling for these multiple factors, along with ethical issues surrounding this research and a tendency towards small sample sizes, a clear conclusion is difficult to make. Other mechanisms by which the use of IV drugs can predispose a person to stroke include cardiac abnormalities, such as arrhythmias and endocarditis, both of which allow for a vegetation or thrombus to form within the heart that can embolize to the circulation and cause a stroke.

The patient presented had a history of IV drug use, but had no evidence of recent use of IV drugs and did not report using cocaine. Routine Holter monitoring did not reveal any significant arrhythmias, and she had no stigmata, symptoms, or vegetations on echocardiography suggestive of endocarditis. Given this clinical picture, the use of cocaine or other IV drugs as a potential cause of her stroke was not considered further. However, she was on methadone maintenance therapy and continued to use marijuana three times daily.

Cannabis use does appear to be a risk factor for stroke, though the strength of this relationship is limited by similar confounding factors as discussed above for cocaine. Many case reports do however suggest a causal link between the two. ${ }^{8}$ Recent evidence supports the potential mechanism that tetrahydrocannabinol (THC), the active ingredient in cannabis, increases oxidative stress and induces cerebral mitochondrial respiratory chain dysfunction, increasing users' potential for stroke. ${ }^{9}$ The relationship between this patient's cannabis use and her stroke is difficult to quantify, as these purported mechanisms have not been readily investigated. Given the lack of clear etiology in this patient's stroke, cannabis use may have been causally related to her ischemic stroke. Unfortunately, a drug screen was not done at the time of the patient's presentation.

\section{Embolic stroke of undetermined source (ESUS)}

The construct of embolic stroke of undetermined source (ESUS) is relatively new, and describes the case in which a patient with confirmed non-lacunar ischemic stroke is not found to have evidence of clinically relevant atherosclerosis, major-risk cardioembolic source (which is excluded using electrocardiogram [ECG] and Holter monitoring to detect atrial fibrillation and with echocardiography to detect intraventricular thrombus), or any other specific causes of stroke, such as migrainerelated stroke, arterial dissection, arteritis, or reversible cerebral artery vasoconstriction syndrome. ${ }^{6}$ Given this definition, this patient fits the criteria for ESUS: she had an MRI-confirmed non-lacunar ischemic stroke, Doppler of her carotid arteries did not reveal 
clinically relevant atherosclerosis, ECG and routine Holter monitoring did not detect atrial fibrillation nor any other cardiac dysfunction. Echocardiography did not show an intraventricular thrombus, though a bubble study looking for a right to left shunt was not done. She had no symptoms of other specific causes of stroke mentioned above, no history of endocarditis, and had neither hypertension nor diabetes, two of the most important modifiable risk factors for stroke. She was found in hospital to have dyslipidemia, with a low-density lipoprotein (LDL) level of $4.5 \mathrm{mmol} / \mathrm{L}$, therefore statin therapy was initiated. She had smoked previously, but had quit two years previous to her stroke, and drank alcohol occasionally. She did not have a family history of premature vascular disease.

The patient's antithrombin III and protein $\mathrm{C}$ were found to be borderline low, however the significance of this is unclear given their only slight abnormality and the context of acute illness during which they were tested. It was recommended that these be repeated in three months' time though the results of this repeat testing are not known. Her rheumatoid factor was also significantly elevated, though she had no symptoms of rheumatoid arthritis. Her cryoglobulin was weakly positive. During her stay in hospital and subsequent to her discharge, she was found to have chronic liver disease associated with Hepatitis $C$. This could account for these abnormalities, as changes to levels of these proteins can be seen in the context of liver disease. Her hypercoagulability screen was otherwise unremarkable: she had normal levels of Lupus anticoagulant, protein S, C3-C4, and anticardiolipin antibodies; her syphilis screen was nonreactive; she did not inherit the most common Factor $\mathrm{V}$ nor prothrombin gene mutations; her ANA was negative; her C-reactive protein was normal though her erythrocyte sedimentation rate was somewhat elevated; and protein electrophoresis did not suggest monoclonal antibodies. Her vasculitis panel was negative. In all, though there were abnormalities present of potential significance, there were no overt causes found for this patient's stroke.

The etiology of ischemic stroke is unknown in about $25 \%$ of all cases, yet is more frequently cryptogenic in younger patients (about one third of cases)., ${ }^{2,3,5,6}$ These estimates vary in large part due to the vague definition of the previously used construct of "cryptogenic stroke", and the lack of specification regarding what extent of diagnostic assessment is necessary before classifying a stroke as "cryptogenic". Though classification systems have previously been developed (e.g., the Trial of Org 10172 in Acute Stroke Treatment [TOAST]),they have tended to have poor inter-rater agreement and inconsistent use has led to high and variable numbers of strokes categorized as cryptogenic (16-39\%).,11
Hence, the need for a well-defined clinical construct to classify these strokes existed, which would allow for better description of the phenomenon and guide research regarding clinical care for these patients. The construct of ESUS will hopefully meet this need.

Potential frequent sources of ESUS thromboembolisms have been explored. Cardiac sources appear to be at play, given that abnormalities are present in many patients with ESUS. However, the absolute risk of these abnormalities causing embolic stroke is low and many are common in the general population (e.g., PFOs and valvular abnormalities); they therefore cannot be said with certainty to have been causational in many cases. ${ }^{6}$ Covert paroxysmal atrial fibrillation (AF) seems to be one of the most important factors in the etiology of ESUS, as a recent study of 2735 patients found that AF was detected during follow up in $29 \%$ of patients with ESUS, and was strongly suspected but not confirmed in a further $14 \% .{ }^{12}$ These episodes can be detected by Holter monitoring or continuous ECG monitoring. Evidence suggests that automated continuous stroke unit ECG monitoring detects significantly more patients with paroxysmal AF than does Holter monitoring. ${ }^{13}$ Furthermore, patients with frequent atrial premature beats detected on 24-hour ECG are at greater risk for paroxysmal AF; hence, these patients are ideal candidates for further prolonged ECG monitoring. ${ }^{14}$ Another important potential cause of ESUS is non-stenotic ( $<50 \%$ diameter stenosis) atherosclerotic carotid artery plaques, which again are common (particularly in elderly populations) and carry a low absolute risk of stroke. ${ }^{6}$ Finally, aortic arch atheroma is commonly found on autopsy, particularly in patients who have succumbed to cerebrovascular disease. This pathology can give rise to emboli, which can travel to the cerebral circulation and cause ischemic stroke. Despite this, imaging of the aortic arch is not routinely done (as in this patient) and this pathology may therefore be under diagnosed. ${ }^{6}$

Research is lacking on the ideal management of ESUS, as it is less often studied than other ischemic stroke subtypes. It seems given the available evidence that patients with ESUS are likely to benefit from anticoagulants, particularly with the reduced risk of intracranial bleeding associated with the use of novel anticoagulants. ${ }^{6}$ Though the merits of antiplatelet agents versus anticoagulants remain unclear, two international, phase III, double-blind, randomized controlled clinical trials (RE-SPECT-ESUS and NAVIGATE ESUS) comparing these two secondary prevention strategies were recently launched. As evidence does not yet support the use of anticoagulant therapy in ESUS, anti-platelet therapy (daily acetylsalicylic acid) was chosen for the patient discussed here. 


\section{Conclusion and follow-up}

This case demonstrated a number of unusual aspects in the care of a stroke patient. Differences in etiology, including the role of illicit drug use, and care for younger stroke patients were discussed. The recently defined construct of ESUS, which occurs more frequently in younger populations, was defined and applied to the patient in question, along with its implications and challenges for secondary prevention. With increasing research in the area, better management strategies may be elucidated.

Just over a month after her initial presentation to the emergency department, the patient was discharged to the Nova Scotia Rehabilitation Center. At the time of writing, she continued to have left-sided deficits, which were slowly improving in rehabilitation.

\section{References}

1. Adams HJ, Davis P, Leira E, Chang K, Bendixen B, Clarke W, et al. Baseline NIH stroke scale score strongly predicts outcome after stroke: a report of the trial of org 10172 in acute stroke treatment (TOAST). Neurology 1999;53(1):126-31.

2. Balci, K, Utku U, Asil T, Celik Y. Ischemic stroke in young adults: risk factors, subtypes, and prognosis. Neurologist 2011;17(1):16-20.

3. Nedeltchev K, A der Maur T, Georgiadis D, Arnold M, Caso V, Mattle H et al. Ischaemic stroke in young adults: predictors of outcome and recurrence. J Neurol Neurosurg Psychiatry 2005;76(2):191-5.
4. Ferro J, Massaro A, Mas JL. Aetiological diagnosis of ischaemic stroke in young adults. Lancet Neurol 2010;9(11):1085-96.

5. Griffiths D, Sturm J. Epidemiology and etiology of young stroke. Stroke Res Treat 2011;2011:209370.

6. Hart R, Diener HC, Coutts S, Easton JD, Granger CB, O'Donnell MJ et al. Embolic strokes of undetermined source: the case for a new clinical construct. Lancet Neurol 2014;13(4):429-38.

7. Sordo L, Indave B, Barrio G, Degenhardt I, de la Fuente L, Bravo M. Cocaine use and risk of stroke: a systemaic review. Drug Alcohol Depend 2014;142:1-13.

8. Hackam D. Cannabis and stroke: systematic appraisal of case reports. Stroke 2015;46(3): 852-6.

9. Wolff V, Schlagowski A, Rouyer O, Charles A, Singh F, Auger C et al. Tetrahydrocannabinol induces brain mitochondrial respiratory chain dysfunction and increases oxidative stress: a potential mechanism involved in cannabis-related stroke. Biomed Res Int 2015;2015:323706.

10. Sloan M, Kittner S, Feeser B, Gardner J, Epstein A, Wozniak M et al. Illicit drug-associated ischemic stroke in the Baltimore-Washington Young Stroke Study. Neurology 1998;50(6):1688-93.

11. Adams H, Bendixen B, Kappelle L, Biller J, Love B, Gordon D et al. Classification of subtype of acute ischemic stroke. Definitions for use in a multicenter clinical trial. TOAST. Trial of Org10172 in Acute Stroke Treatment. Stroke 1993;24(1):35-41.

12. Ntaios G, Papavasileiou V, Milionis H, Makaritsis K, Manios E, Spengos K et al. Embolic stroke of undetermined source in the Athens Stroke Registry: a descriptive analysis. Stroke 2015;46(1)176-81.

13. Rizos T, Guntner J, Jenetzky E, Marquardt L, Reichard C, Becker R et al. Continuous stroke unit electrocardiographic monitoring versus 24-hour holter electrocardiography for detection of paroxysmal atrial fibrillation after stroke. Stroke 2012;43(10):2689-94.

14. Wallmann D, Tuller D, Wustmann K, Meier P, Isenegger J, Arnold M et al. Frequent atrial premature beats predict paroxysmal atrial fibrillation in stroke patients: an opportunity for a new diagnostic strategy. Stroke 2007;38(8):2292-4.

15. Kittner S, Stern B, Wozniak M. Cerebral infarction in young adults: the Baltimore-Washington Cooperative Young Stroke Study. Neurology 1998;50(4):890-4. 